\title{
Male size, developmental stability, and mating success in natural populations of three Drosophila species
}

\author{
THERESE ANN MARKOW \& JEFFRY P. RICKER \\ Department of Zoology, Arizona State University, Tempe, AZ 85287-1501, U.S.A.
}

\begin{abstract}
Morphological correlates of male mating success were assessed in natural populations of three Drosophila species. Mating males in $D$. simulans were larger than single males but were characterized by reduced developmental stability as indicated by fluctuating asymmetry. Mating male $D$. pseudoobscura were no larger than single males but exhibited significantly greater developmental stability. In $D$. mojavensis, however, mating males were larger and they showed a similar level of fluctuating asymmetry to that observed in single males. The differences observed between species are discussed in the context of their mating systems and reproductive ecology.
\end{abstract}

Keywords: Drosophila, fluctuating asymmetry, sexual selection.

\section{Introduction}

Male mating succcess in Drosophila has been extensively studied in the laboratory and appears to be related to male size for a number of species (Partridge et al., 1987a; Markow, 1985; Markow, 1987b; Markow, 1988). Very few studies of Drosophila male mating success have been conducted in the field but those which have been reported also support the importance of male size in D. melanogaster (Partridge et al., 1987a; Markow, 1988), D. nigrospiracula (Markow, 1988), D. buzzatii (Santos et al., 1988) and D. testacea (James \& Jaenike, 1991). The relationship is less consistent for D. pseudoobscura observed at baits in that not all replicates showed an advantage for large males (Partridge $e t$ al., 1987b), which suggests that in some species directional selection for male size may not always be associated with mating success.

A number of investigations report a reduction in fitness of individuals whose phenotypes lie towards the ends of distributions, supporting the action of balancing or stabilizing selection (Lerner, 1954; 1958). The extent to which balancing sexual selection occurs in natural Drosophila populations has not been examined. However, body size is a quantitative trait, and individuals much larger or smaller than the population average are expected to show reduced developmental homeostasis due to homozygosity at the loci contributing for their large or small phenotypes. Thus the prediction follows that if successfully mating males are signifi- cantly larger, they should also tend to exhibit less developmental stability. A contrasting prediction is that if there is balancing sexual selection on male size, it should be the developmentally stable, average size males who experience the greatest success as these males are closest to the population average.

Developmental stability has been measured in Drosophila but never as it relates to mating success. The most widely employed measure of developmental homeostasis is fluctuating asymmetry, or the difference in magnitude of a trait between the right and left side (Palmer \& Strobeck 1986). The bilateral traits most often used to calculate fluctuating asymmetry in Drosophila have been numbers of bristles, especially the sternopleurals (Reeve, 1960; Van Valen, 1962: Parsons, 1962; Markow \& Ricker, 1991). However, any paired structure may show fluctuating asymmetry.

The present study sought to accomplish several things. First, we wished to assess potential correlates, in addition to male size, of mating success in order to gain a more complete understanding of sexual selection in nature. Second, we wished to expand the existing database on mating behaviour in natural Drosophila populations to include additional species. In the present study, we asked if mating males could be distinguished from non-mating males by their size or developmental stability in several species. We calculated fluctuating asymmetry for several independent measures, wing length and regional bristle numbers, to examine the relationship between developmental 
stability and mating success in three Drosophila species.

Three unrelated species were selected for study: $D$. simulans, D. pseudoobscura, and D. mojavensis. Drosophila simulans is the sibling species of $D$. melanogaster with whom it is often sympatric. In many Tempe, Arizona citrus groves, $D$. simulans are more common than $D$. melanogaster, although both may be collected from the same piece of fruit. At the same localities, $D$. pseudoobscura are found in smaller numbers throughout the winter and spring. The last species, D. mojavensis is endemic to the Sonoran Desert and utilizes necrotic arms of columnar cacti to feed and reproduce. These three species were selected because their mating systems are under investigation in our laboratory and because they are frequently discussed in the genetics, evolution, and behaviour literature.

\section{Methods}

\section{Field observations}

Drosophila simulans and D. psuedoobscura were observed on fallen citrus (lemons and grapefruit) in Tempe, Arizona. The distribution of males and females on different types of fallen citrus was assessed on three different days. Fruit types were classified as in Markow (1988) as being newly fallen and cracked open exposing the citrus flesh, fallen but with the skin intact, or decaying. Drosophila simulans was the predominant species, with $D$. pseudoobscura the next most common. Occasional D. melanogaster males were collected but discarded from the samples. Mating activity shows two peaks, one in the morning, the other just prior to dusk (Markow, 1987a). Flies were collected at both activity periods for about 1 week during May of both 1988 and 1989. As soon as a mating pair was observed, it was aspirated into a vial and a single male was immediately aspirated from the same area into another vial. Flies were taken back to the lab- oratory each day to be measured for thorax length and for wing mounting (see below).

Drosophila mojavensis were observed on their natural host plant, organpipe cactus (Stenocereus thurberi) at our long-term study site in the desert near San Carlos, Sonora, Mexico (Markow \& Toolson 1990). Three different observations/collection periods were employed on three different cacti in March 1989. Distribution of males and females at various locations on necrotic cacti were reported previously in Markow \& Toolson (1990). As with the other two species, when a copulating pair was observed, it was aspirated into a vial and a single male from nearby was immediately aspirated into a second vial. Thorax lengths were measured in Mexico. Wings were prepared for measuring in Mexico but returned to the laboratory in Tempe.

\section{Measurements on flies}

Thorax lengths were measured under a dissecting microscope with an ocular micrometer. Several other bilateral traits were measured. Wing lengths were measured for males of both species. Wings were removed with forceps after thorax lengths were recorded and placed on double-sided transparent tape that had been affixed to a clear $35-\mathrm{mm}$ projection slide. Wings were projected onto a wall covered with paper for measuring. The length measured was from the anterior crossvein to the end of the second longitudinal vein (Fig. 1) and was in arbitrary units. Although the position of the projector with respect to the wall was marked on the floor in order to be consistent in measurements between years, a seemingly imperceptible change in the position set-up in the second year caused some slight differences in the wing measurements between years. In the second year, additional traits were added to those measured for $D$. simulans and $D$. pseudoobscura. These included the number of sternopleural bristles, orbital and frontal bristles, and aristal branches (Van
Fig. 1 Line for wing length measure extended from the anterior crossvein to the end of the second longitudinal vein.

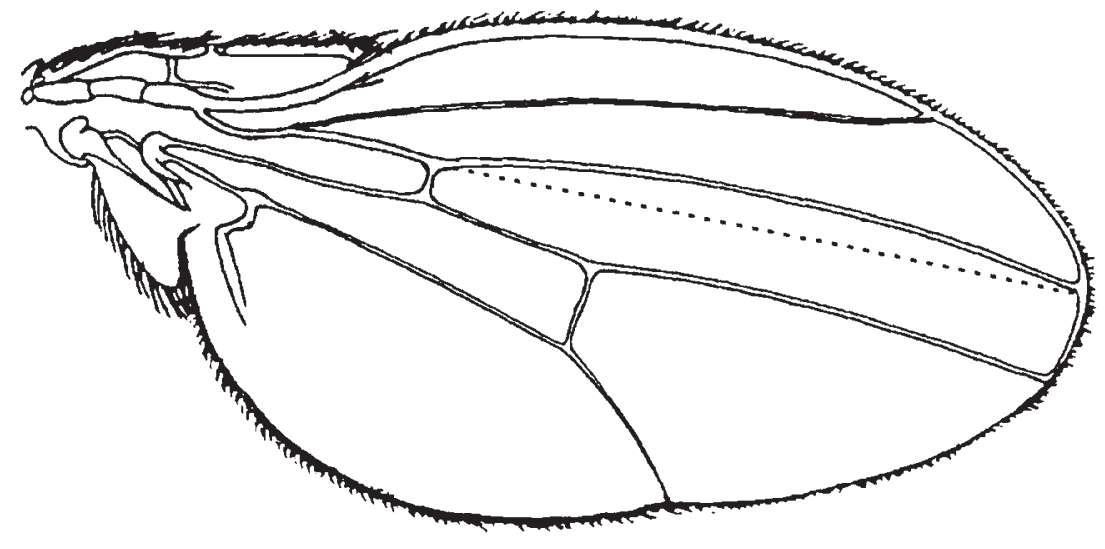


Valen, 1962), all counted under a dissecting microscope. The dark coloration interfered with out ability to count sternopleurals or orbital bristles in $D$. pseudoobscura.

\section{Analysis of fluctuating asymmetry (FA)}

Asymmetry scores were calculated by subtracting the value of the left side from that of the right for each of the traits. Several factors are known to confound analysis of fluctuating asymmetry (Palmer \& Strobeck, 1986) and must be tested for and corrected if they exist. One is the existence of a positive correlation between the magnitude of the trait and the degree of asymmetry. The other is the presence of directional asymmetry. In one species, $D$. pseudoobscura, wing length and wing length asymmetry were correlated $(r=0.46 . P<0.01)$, which was corrected for by dividing each individual $(R-L)$ value by the average magnitude of the trait $(R+L / 2)$. Directional asymmetry $(D A)$ was measured by factorial ANOVA using side as a factor. None of the traits were found to exhibit DA in any species. When the factor 'male-type' (i.e. single or mating) shows statistical significance, a group difference in FA is indicated.

\section{Results}

\section{Drosophila simulans}

Sex ratios differ dramatically on each of the three fruit types (Table 1). On the exposed flesh of fruit that had recently broken open, females significantly outnumbered males. It was easy to observed females ovi-

Table 1 Distributions of flies collected on flesh of crackedopen fruit, skin of intact fruit, and decayed fruit. For both species, data from different days were tested for homogeneity prior to pooling

\begin{tabular}{|c|c|c|c|c|c|}
\hline & \multicolumn{2}{|c|}{ Females } & \multicolumn{2}{|c|}{ Males } & \multirow[b]{2}{*}{$\chi^{2}(1: 1)$} \\
\hline & $n$ & $\%$ & $n$ & $\%$ & \\
\hline \multicolumn{6}{|l|}{ D. simulans } \\
\hline Flesh & 356 & 65.3 & 189 & 34.7 & 51.17 \\
\hline Skin & 201 & 42.0 & 277 & 58.0 & 12.08 \\
\hline Decayed & 124 & 54.1 & 105 & 45.9 & 1.58 \\
\hline Total & 681 & 54.4 & 571 & 45.6 & 2.62 \\
\hline \multicolumn{6}{|c|}{ D. pseudoobscura } \\
\hline Flesh & 85 & 52.8 & 76 & 47.2 & 0.50 \\
\hline Skin & 31 & 50.1 & 30 & 49.9 & 0.02 \\
\hline Decayed & 44 & 39.3 & 68 & 60.7 & 5.14 \\
\hline Total & 160 & 47.9 & 174 & 52.1 & 0.59 \\
\hline
\end{tabular}

positing on this class of fruit, and within a day, the fruit surface was usually plastered with eggs. Few copulations were observed on fruits of this type. There was a slight but significant male bias on the skin of intact fruit. The slight female bias on decaying fruit was not significant. Flies were observed to be feeding on the microbial flora on the fruit in this class. There was considerable courtship activity among flies on decayed fruit. Males would court any female encountered. Most of the copulations were observed on pieces of decaying fruit, although for the total number of flies present and the level of courtship activity, copulations were rare which suggests that most females were unreceptive.

Mating and single males that were used for measurements were all collected from decaying fruit (Table 2). Males caught in copula in both years, were longer in the thorax. Wing lengths of mating males were greater only in the first year. The length of the thorax of all flies was larger in the second year but the difference in wing length between the 2 years was due to the artefact described in the methods section. Wing length and thorax length were highly correlated $(r=0.7669, P<0.0001)$. Single and mating males did not differ in the additional quantitative characters measured in the second year: aristal branch number, head bristle number, or sternopleural bristle number. There was no relationship between wing length and wing length fluctuating asymmetry in this species $\langle r=0.18961, P=0.1872\rangle$. Fluctuating asymmetry was always greatest in the copulating males but the difference was significant only for wing length and aristal branching. The direction of wing fluctuating asymmetry differences for male type was consistent in both years. None of the measures of fluctuating asymmetry were correlated. Thus copulating males of $D$. simulans were larger and, as such, exhibited the expected increase in fluctuating asymmetry.

\section{Drosophila pseudoobscura}

Far fewer flies of $D$. pseudoobscura than of $D$. simulans were at the site. While a large number of $D$. simulans remained on fallen fruit throughout the day and night (Markow, 1987a), D. pseudoobscura came to the fruit for only about $1 \mathrm{~h}$ in the morning and again in the early evening. The distribution of males and females (Table 1) reveals that the only departure from a 1:1 sex ratio was on the decayed fruit where it was significantly male-biased. As in $D$. simulans the majority of copulations occurred on the decaying fruit. $D$. pseudoobscura of both sexes were also observed to feed on the decaying fruit but females were never observed ovipositing. The behaviour of $D$. pseudoobscura males shows some obvious differences with $D$. 
Table 2 Characteristics of single and mating males in a natural population of $D$. simulans

\begin{tabular}{lcc}
\hline & $\begin{array}{l}\text { Single male } \\
(n=50)\end{array}$ & $\begin{array}{c}\text { Copulating male } \\
(n=50)\end{array}$ \\
\hline 1988 & & \\
Thorax length & $0.8743 \pm 0.01$ & $0.9049 \pm 0.01^{*}$ \\
Wing length & $48.89 \pm 0.71$ & $51.61 \pm 0.67^{*}$ \\
Wing length FA & $0.380 \pm 0.07$ & $0.560 \pm 0.09$ \\
1989 & & \\
Thorax length & $0.9481 \pm 0.01$ & $0.9653 \pm 0.01^{*}$ \\
Wing length & $46.75 \pm 0.60$ & $46.16 \pm 0.57$ \\
Wing length FA & $0.689 \pm 0.04$ & $0.93 \pm 0.04^{*}$ \\
Aristal branches & $7.3100 \pm 0.18$ & $7.300 \pm 0.17$ \\
Aristal branch FA & $0.9000 \pm 0.12$ & $1.2000 \pm 0.12^{*}$ \\
Head bristles & $9.8617 \pm 0.09$ & $9.6800 \pm 0.08$ \\
Head bristle FA & $0.3617 \pm 0.09$ & $0.4800 \pm 0.08$ \\
Sternopleural bristle & $12.9100 \pm 0.23$ & $13.20 \pm 0.21$ \\
Sternopleural bristle FA & $1.1000 \pm 0.15$ & $1.20 \pm 0.15$ \\
\hline
\end{tabular}

$* P<0.05$.

simulans. Males were seen to stand in isolation and scissor both wings creating a highly reflectant flashing. They were seen to perform the 'flashing' behaviour for up to 15 min despite the absence of other conspecifics nearby. Males also exhibited aggression to other males on the same piece of fruit. Although there were far fewer $D$. pseudoobscura at the site than $D$. simulans, a higher proportion were found copulating.

As in $D$. simulans, wing and thorax lengths were highly correlated $(r=0.6965, P<0.0001)$. However, no difference was observed between copulating and single males for thorax length, wing length or aristal branch number in either year (Table 3). Wing fluctuating asymmetry was significantly greater in the single male category in both years. No difference in aristal branch FA was detected in the 1 year it was measured. Thus, in $D$. pseudoobscura males, greater developmental stability rather than size was associated with mating success.

\section{Drosophila mojavensis}

As reported earlier by Markow \& Toolson (1990), matings tended to occur on cactus arms away from necrotic ooze. Females were observed to fly to these sites after males had settled at them and the movement of the arriving females triggered males to court them. Many matings were observed, although the spiny setting was not always conductive to apsirating flies. The only difference between mating and single males in this species was in thorax length, which was greater in mating males (Table 4). Curiously, thorax length and wing length were uncorrelated $(r=0.3201, P=0.184)$.
Table 3 Characteristics of single and mating males in a natural populations of $D$. pseudoobscura

\begin{tabular}{lcc}
\hline & Single male & Copulating male \\
\hline 1988 & & \\
Thorax length & $1.0377 \pm 0.0108$ & $1.0356 \pm 0.0124$ \\
Wing length & $67.45 \pm 0.667$ & $68.35 \pm 0.772$ \\
Wing length FA & $0.660 \pm 0.097$ & $0.050 \pm 0.086^{*}$ \\
1989 & & \\
Thorax length & $1.0855 \pm 0.0091$ & $1.0834 \pm 0.0115$ \\
Wing length & $59.855 \pm 0.4811$ & $60.011 \pm 0.7762$ \\
Wing FA & $0.1111 \pm 0.0473$ & $0.0217 \pm 0.0217^{*}$ \\
Aristal branches & $7.1111 \pm 0.0708$ & $7.2282 \pm 0.7725$ \\
Aristal branch FA & $0.4440 \pm 0.0813$ & $0.3261 \pm 0.6988$ \\
\hline
\end{tabular}

$* P<0.05$.

Mating and single males did not differ in fluctuating asymmetry.

\section{Discussion}

Mating males were larger than single males in two of the three species. Each species showed a different pattern of association between mating status and morphological phenotype. Mating males were larger in $D$. simulans and showed reduced developmental stability, in keeping with the first prediction. Single and mating $D$. pseudoobscura males were the same size but mating males were characterized by greater developmental stability, which was consistent with the second prediction. The observations on $D$. mojavensis, how- 
Table 4 Characteristics of single and mating males from a natural population of $D$. mojavensis

\begin{tabular}{lccc}
\hline & Single males (22) & Mating males (22) \\
\cline { 1 - 1 } Thorax length & $0.879 \pm 0.009$ & $0.923 \pm 0.008^{*}$ \\
Wing length & $27.28 \pm 0.344$ & $28.20 \pm 0.402$ \\
Wing FA & $0.905 \pm 0.209$ & $0.895 \pm 0.263$ \\
SP bristles & $26.545 \pm 0.491$ & $26.364 \pm 0.743$ \\
Bristle FA & $1.273 \pm 0.239$ & $1.545 \pm 0.235$ \\
\hline
\end{tabular}

$* P<0.05$.

ever, were unexpected. Mating males were larger than single males but no other differences were apparent. The observed absence of any correlations for fluctuating asymmetry in different characters was expected, based upon earlier work (Van Valen, 1962).

Each of the three species examined here differ dramatically in their mating systems and ecology and these differences must ultimately influence the nature and intensity of sexual selection. The distribution of male and female $D$. simulans on fallen fruit is very similar to that reported by Markow (1988) for its sibling species $D$. melanogaster. There was no size difference for $D$. melanogaster between mating and single males found on decaying fruit. Males collected from the skin of unbroken pieces of fruit were significantly smaller, however, suggesting that male size is important in determining who has access to the sites where receptive females are feeding. Male size in D. simulans is clearly an important determinant of which males on the same piece of fruit actually mate. Copulating males are not only larger but as a group show a decrease in developmental stability. This observation is consistent with the existence of directional sexual selection on size or some other quantitative trait. It is likely, however, that the advantage of large size for mating is countered by opposing selection on development time as described by Wilkinson (1987) in D. melanogaster. Female oviposition behaviour supports the idea that rapid development is advantageous. We observed females ovipositing immediately on fresh fallen fruit and it is clear from the speed at which the fruit decomposes that rapid oviposition is necessary in order for larval development to finish before the fruit dries up.

Drosophila pseudoobscura males who were found mating were no larger on average than single males but showed superior developmental stability as reflected in wing length FA. There may be certain conditions under which larger males experience an advantage, as Partridge et al. (1987b) found in approximately half of their collections on bait buckets that mating males were significantly larger. Baits are likely to provide a different sort of arena for sexual selection than pieces of fruit. In addition, populations may have different age structures on different days and male age is known to influence mating success (Long et al., 1980). Judging from the excess of males at the mating sites and the 'flashing' behaviour of males, it may be that sexual selection acts more strongly on certain wing attributes than on overall size. For example, wing symmetry may be important in the ability to deliver some signal to other males or to females.

Drosophila mojavensis male size may confer some advantage in male-male competition within the lek where the sex ratio is highly male biased (Markow \& Toolson, 1990). However, the larger males show no decrease in developmental stability. Unlike the other species, males in $D$. mojavensis are not sexually mature until they are 8-days-old (Markow, 1982), thus natural selection has a longer opportunity, compared to the other two species, to operate on males. The Sonoran desert is a harsh environment and all males who exhibit developmental instability may not reach the age of reproductive maturity. Large size may also confer an advantage in reducing water loss (Markow \& Toolson, 1990 ), providing females with some indication of relative male quality. Receptive females come to male arenas located away from feeding sites and oviposition sites. As they are coming to mate and not to feed and oviposit, they are different from the population of feeding females encountered by $D$. simulans or $D$. pseudoobscura males. Additional and important differences exist between $D$. mojavensis and the other two species. Daily female remating (Markow, 1982) increases mating opportunities and the significant male ejaculatory investment (Markow \& Ankney, 1984) and mate discrimination by males (Markow, 1982) suggest that the nature and intensity of sexual selection are very different in $D$. mojavensis.

Reduced size variability in mating compared to single males has been reported for Drosophila buzzatii (Santos et al., 1988; Ruiz et al., 1991), D. sylvestris (Boake, 1989) and D. melanogaster (Markow \& Sawka 1991), which suggests that stabilizing selection has a role in courtship success in these species. The data presented here are also compatible with the action of balancing sexual selection. In all three species examined in the present study, the largest as well as the smallest males were found in the single male groups.

Note that field studies differ from laboratory experiments in several respects. We cannot control in nature for male age or mating experience, both of which are known to influence male success (Markow, 1987). In addition, laboratory experiments are typically conducted with virgin females. We know from earlier studies (Gromko \& Markow, 1991; T. Markow, unpublished observations) that nearly 100 per cent of females 
in natural populations of these species are already inseminated at any given time and that the majority of observed copulations, which are infrequent considering the large number of flies at the breeding sites, represent rematings by females. It is possible, then, that the differences we detect between mating and single males are important for rematings but that virgin matings, if they could be selectively observed, might reveal a different pattern of factors associated with male success.

Finally, the resource ecology of these three species may vary seasonally or with different hosts, creating different suites of selective forces on characters related to mating success. Just as caution should be used in extrapolating from laboratory experiments to natural populations, observations in nature will be most informative if made in more than one setting.

\section{References}

BOAKE, C. 1989. Correlation between courtship success, aggressive success, and body size in a picture singed fly, Drosophila silvestris. Ethology, 80, 318-329.

GROMKO, M. H. AND MARKow, T. A. 1991. Courtship and remating in field populations of Drosophila. Anim. Behav., (in press).

JAMES, A. AND JAENIKE, J. 1991. Determinants of male mating success in Drosophila tectacea. Anim. Behav., (in press).

LERNER, 1. M. 1954. Genetic Homeostasis. John Wiley, New York.

LERNER, I. M. 1958. The Genetic Basis of Selection. John Wiley, New York.

LONG, C., MARKOW, T. AND YAEGER, P. 1980. Relative male age, fertility, and competetive mating success in Drosophila melanogaster. Behav. Genet., 10, 163-170.

MARKOW, T. 1982. Mating systems of Cactiphilic Drosophila. In: Barker, J. S. F. and Starmer, W. T., (eds) Ecological Genetics and Evolution: the Cactus-Yeast-Drosophila Model System. Academic Press, New York.

MARKow, T. A. 1985. A comparative investigation of the mating system of Drosophila hydei. Anim. Behav., 33, 775-781.

MARKow, T. 1987a. Nocturnal emissions in Drosophila simulans. Drosophila Information Service. 66, 95.

markow, T. A. 1987b. Genetics of sexual selection in Droso- phila. In: Huettle, M. (ed.) Genetics of Invertebrate Behavior. Plenum. New York.

MARKOW, T. 1988. Reproductive behavior of Drosophila in the laboratory and in the field. J. Comp. Psychol., 102, 169-174.

MARKOW, T. AND ANKNEY, P. 1984. Drosophila males contribute to oogenesis in a multiple mating species. Science, 224, 302-303.

MARKOW, T. A. AND RICKER, J. 1991. Developmental stability in hybrids between the sibling species pair Drosophila melanogaster and Drosophila simulans. Genetica, 84, 115-121.

MARKoW, T. AND SAWKA, s. 1991. Dynamics of sexual selection in experimental groups of Drosophila melanogaster. (in press).

MARKOW, T. AND TOOLSON, E. 1990. Temperature shift, epicuticular hydrocarbons and sexual isolation in Drosophila mojavensis. In: Starmer, W. T. and Barker, J. S. F., (eds) Ecology and Evolutionary Genetics of Drosophila. Plenum, New York.

PALMER, A. R. AND STROBECK, C. 1986. Fluctuating asymmetry: Measurement, analysis, patterns. Ann, Rev. Ecol. Syst., 17, 291-321.

PARSONS, P. A. 1962. Maternal age and developmental variability. J. Exp. Biol., 39, 251-260.

PARTRIDGE, L., EWING, E. AND CHANDLER, A. 1987a. Male size and mating success in Drosophila melanogaster. The role of female and male behaviour. Anim, Behav., 35, 555-562.

PARTRIDGE, L., HOFFMANN, A. AND JONES, s. $1987 \mathrm{~b}$. Male size and mating success in Drosophila melanogaster and Drosophila pseudoobscura under field conditions. Anim. Behav., 35, 468-476.

REEVE, E. C. R. 1960. Some genetic tests on asymmetry of sternopleural bristle number in Drosophila. Genet. Res., 1, 151-172.

RUIZ, A., SANTOS, M., BARBADILLA, A., QUEZADA-DIAZ, J. E., HASSON, E. AND FONTEDEVILA, A. 1991. Genetic variance for body size in a natural population of Drosophila buzzatii. Genetics, 128, 739-750.

SANTOS, M., RUIZ, A., BARBADILLA, A., QUEZADA-DIAZ, J. E., HASSON, E. AND FONTEDEVILA, A. 1988. The evolutionary history of Drosophila buzzatii XIV. Larger flies mate more often in nature. Heredity, 61, 255-262.

VAN VALEN, L. 1962. A study of fluctuating asymmetry. Evolution, 16, 125-141.

WILKINSON, G. s. 1987. Equilibrium analysis of sexual selection in Drosophila melanogaster. Evolution, 41, 11-21. 